\title{
Time Variations of the Modulation of Anomalous and Galactic Cosmic Rays
}

\author{
E.R. Christian ${ }^{1}$, W.R. Binns ${ }^{2}$, C.M.S. Cohen ${ }^{3}$, A.C. Cummings ${ }^{3}$, J.S. George ${ }^{3}$, P.L. \\ Hink $^{2}$, J. Klarmann ${ }^{2}$, R.A. Leske ${ }^{3}$, M. Lijowski ${ }^{2}$, R.A. Mewaldt ${ }^{3}$, P.L. Slocum ${ }^{4}$, \\ E.C. Stone ${ }^{3}$, T.T. von Rosenvinge ${ }^{1}$, M.E. Wiedenbeck ${ }^{4}$, and N. Yanasak ${ }^{4}$ \\ ${ }^{1} N A S A /$ Goddard Space Flight Center \\ ${ }^{2}$ Washington University, St. Louis \\ ${ }^{3}$ California Institute of Technology \\ ${ }^{4}$ Jet Propulsion Laboratory
}

\begin{abstract}
Between the launch of the Advanced Composition Explorer (ACE) in 1997 and the end of 1999, the intensities of galactic cosmic rays at $1 \mathrm{AU}$ have dropped almost a factor of 2 , and the anomalous cosmic rays have decreased by an even larger amount. The large collecting power of the Cosmic Ray Isotope Spectrometer (CRIS) and the Solar Isotope Spectrometer (SIS) instruments on ACE allow us to investigate the changing modulation on short time scales and at different rigidities. Using anomalous cosmic ray (ACR) and galactic cosmic ray (GCR) intensities of He, $\mathrm{C}, \mathrm{O}, \mathrm{Ne}, \mathrm{Si}, \mathrm{S}$, and $\mathrm{Fe}$, and energies from $-6 \mathrm{MeV} /$ nucleon to $\sim 460 \mathrm{MeV} /$ nucleon, we examine the differences between the short term and long term effects. We observe the expected correlation of these intensities with neutron monitor data, but see little correlation of GCR and ACR intensities with the locally measured magnetic field.
\end{abstract}

\section{INTRODUCTION}

Although modulation of cosmic, rays by the heliospheric magnetic field has been studied and modeled for a long time, the exact roles of drifts, global merged interaction regions (GMIRs), and slow variations in the transport parameters is still unresolved (see, e.g., (1) and references therein). The launch of the Advanced Composition Explorer (ACE) in August 1997 has brought new tools to bear, including large, high-resolution spectrometers such as the Solar Isotope Spectrometer (SIS) (2) and the Cosmic Ray Isotope Spectrometer (CRIS) (3), as well as in-situ heliospheric solar wind and magnetic field measurements.

In this work, we examine data taken with both ACE, situated at the L1 Earth-Sun libration point, and an Earth-based neutron monitor. The data spans approximately two years, from late 1997 to late 1999 .

\section{OBSERVATIONS}

Using SIS and CRIS data that is available on the web from the ACE Science Center (http://www.srl. caltech.edu/ACE/ASC/level2/index.html), we have begun to study short and long term variations in the modulation of both ACRs and GCRs. Twenty-eight different elemental species and energy bins were used, picked so that the intensities were dominated by either ACRs or GCRs for the entire time period studied here. Three ACR intensities from SIS were used: He 6-10 $\mathrm{MeV} /$ nucleon; O 7-13 MeV/nucleon; and O 13-21 $\mathrm{MeV} /$ nucleon. SIS measurements of intensities for carbon (33-76 MeV/nucleon), silicon (53-123 $\mathrm{MeV} /$ nucleon), and iron (70-168 MeV/nucleon) are included in the GCR data set since ACR contributions for these combinations of charge and energy should be negligible. At CRIS energies, the data is dominated by GCRs and four energy bins each were used from carbon, oxygen, neon, silicon, and 


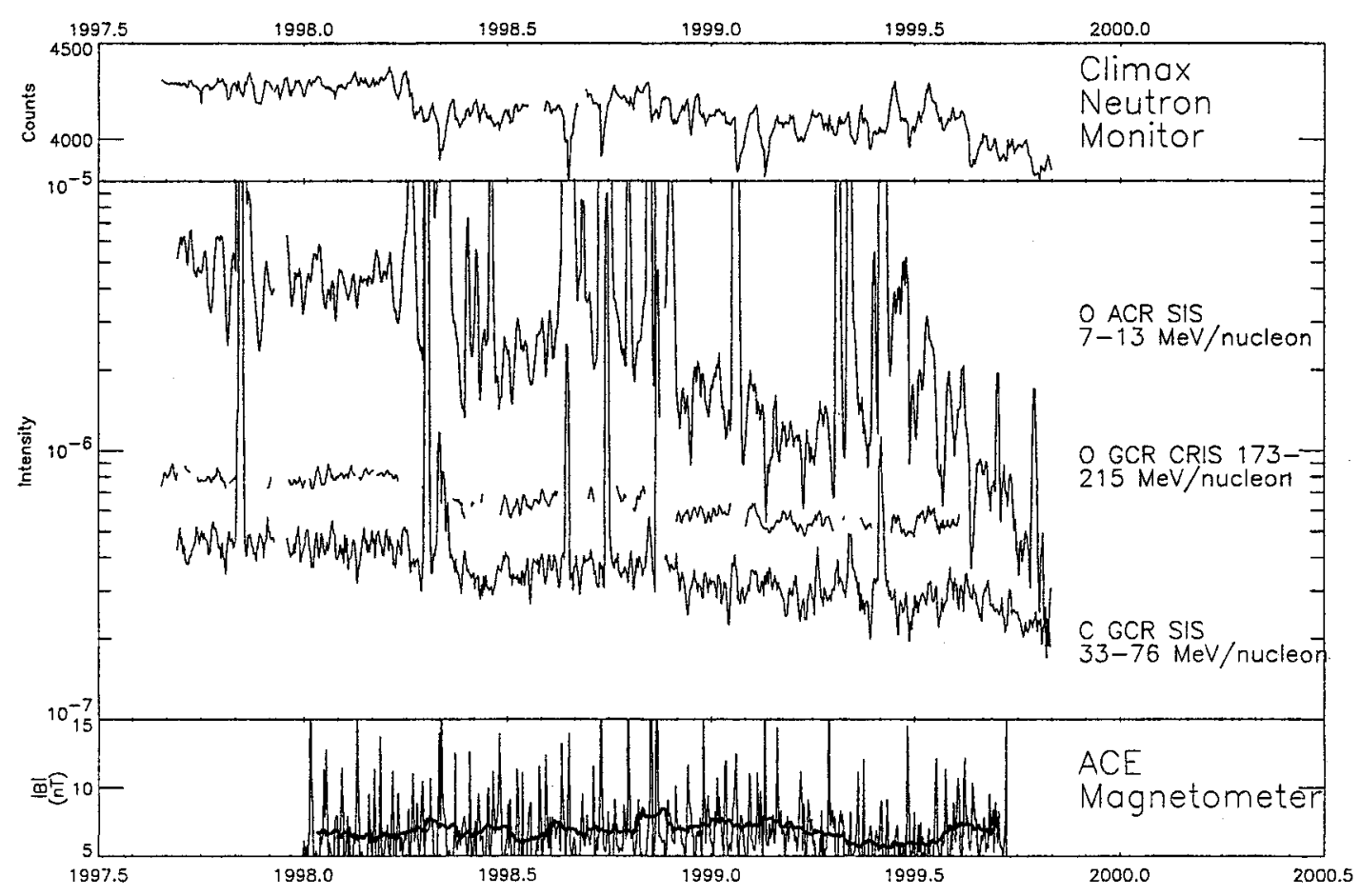

FIGURE 1. Top panel: time profiles for the Climax neutron monitor daily count rate. Middle panel: selected particle intensities (particles/ $\mathrm{cm}^{2} \mathrm{sr}$ sec MeV/nucleon); 3-day running average ACR Oxygen between 7 and $13 \mathrm{MeV} /$ nucleon from SIS (+), daily average GCR Oxygen, 173-215 MeV/nucleon from CRIS (solid circle), and 3-day running average GCR Carbon 33-76 $\mathrm{MeV} /$ nucleon from SIS (x). Bottom panel: magnetic field amplitude from ACE MAG, shown as both daily averages (thin line) and a 27-day running average (thick line).

iron, and two from sulfur, ranging from 60 $\mathrm{MeV} /$ nucleon carbon to $460 \mathrm{MeV} /$ nucleon iron.

Figure 1 shows the daily averages of three of these intensities in the middle panel. Gaps in the CRIS data set are because the CRIS instrument was not designed to work during solar active periods. The top panel shows the Climax neutron monitor daily count rate, and the bottom panel shows the ACE/MAG (4) magnetic field magnitude as both daily average and 27-day running averages. The increase in modulation since the middle of 1997 is obvious in the neutron monitor data and in the ACRs and GCRs. The 27-day magnetic field averages have remained remarkably flat during this same period.

\section{DISCUSSION}

The intensity levels early in this time period appear to have decreased in a step-wise fashion, where the steps (see e.g. April 1998) were coincident with increases in solar activity, as has been previously reported (see, e.g., 5,6). However, these apparent steps may be due to medium-term modulation events with recovery ("Forbush decreases") superimposed on the slowly increasing modulation level. Since about the middle of 1999, the increase in modulation has been gradual.

Figure 2 shows the correlation between the highenergy neutron monitor data and ACE intensities for two of the twenty-eight data sets used in this study. In order to improve statistics, each energy bin was averaged for a time period that varied between 1 day and 14 days, depending upon flux. From the straight line fit on the log-log plot, the power law index, $\alpha$, of the correlation is derived. It has been previously shown ((7) and references therein), that ACR O intensities are well correlated with neutron monitor data taken to the thirtieth power, and that the GCR are correlated with power law index of $\sim 6-7$ (8). The indices calculated in this work (Figure 3) are consistent with earlier GCR indices and slightly lower for ACRs when compared with (7). 
In Figure 3, the power law indices are plotted versus the median rigidity for each data set. The GCR indices are approximately seven at low rigidities, getting somewhat smaller about $1300 \mathrm{MV}$. This is fully expected because, in the region of the spectra where the intensity is proportional to the kinetic energy ( $\mathrm{J}=\mathrm{AT}(9))$, the intensities all vary together, but above this region, higher rigidity intensities are less affected by changes in modulation. If we had data that extended up to the rigidity of the Climax neutron monitor $(\sim 3 \mathrm{GV})$, presumably the power law index of the correlation would be one.

It has been suggested that fundamental processes at the Sun produce heliospheric magnetic field enhancements which are responsible for cosmic ray modulation ((1), (10), (11) and references therein). This is frequently shown as an anticorrelation between the locally-measured average magnetic field strength and the particle intensities. The current observations are not consistent with this picture, as can be seen in Figure 1, which shows no increase in the $1 \mathrm{AU}$ magnetic field that corresponds to the increase in modulation level.

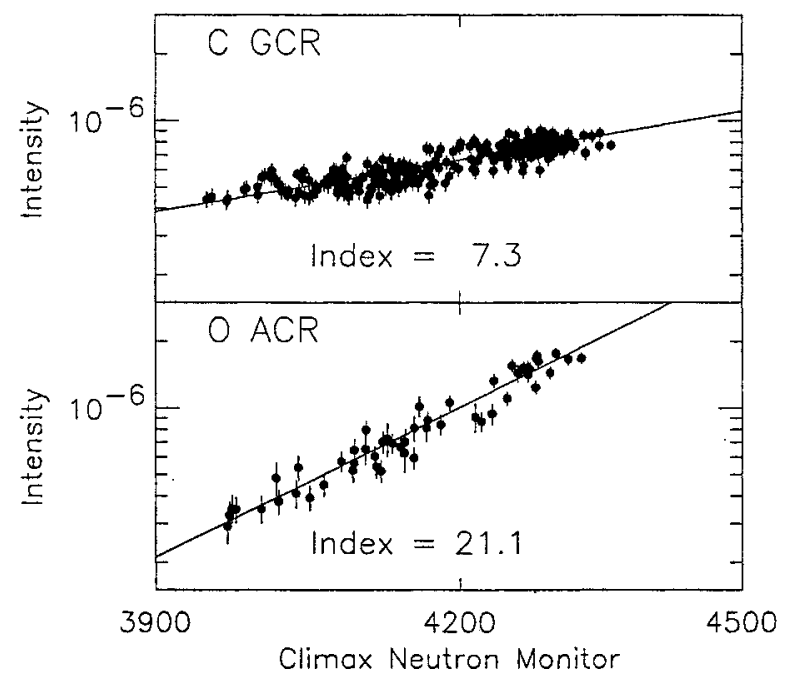

FIGURE 2. Cosmic Ray Intensity vs. Climax neutron monitor counts for (top) GCR Carbon 100-150 MeV/nucleon from CRIS and (bottom) ACR Oxygen 13-21 MeV/nucleon from SIS. The solid lines are the best-fit power laws and the power law indices are shown on each plot. Data in the top panel are daily averages and in the bottom are 7-day averages to improve statistics.

This is shown in a different way in Figure 4 by plotting (for two of the energy bins) the particle intensity versus the average, locally measured magnetic field strength. The data have been averaged over distinct 27-day time periods. The Pearson's correlation coefficient, $r$, is shown on both parts of
Figure 4, and for all 28 data sets varied between 0.2 and -0.3 , indicating that there is no strong correlation, which is inconsistent with the observations of (11), however, their data also includes time periods in which the modulation level was increasing without a corresponding increase in the measured magnetic field. This may be partially due to the use of a local magnetic field strength, which does not always correlate with the overall heliospheric magnetic field.

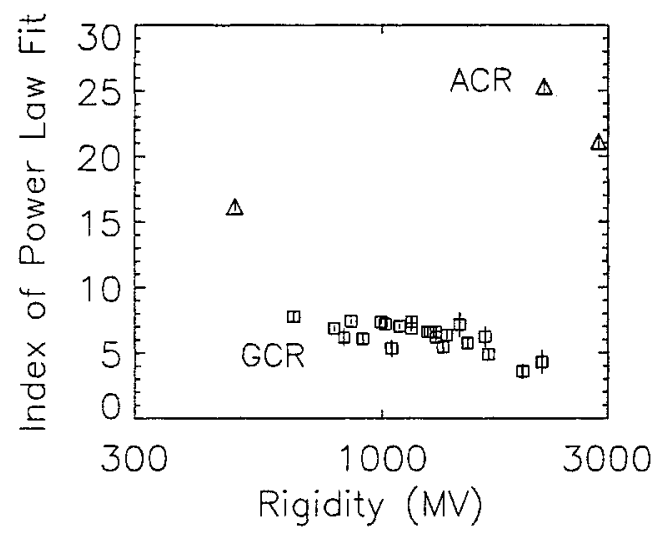

FIGURE 3. Indices of power-law fits between Climax neutron monitor data and ACE particle intensities vs. median rigidity of particle intensities. The triangles are ACR data (charge $=+1$ ), and the squares are GCR data (fully stripped).

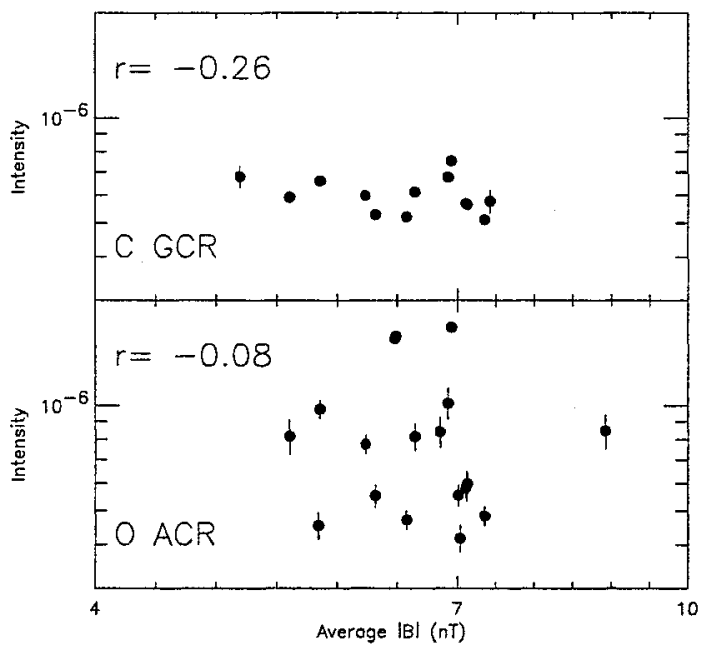

FIGURE 4. Cosmic ray intensity (particles / $\mathrm{m}^{2}$ sr $\mathrm{sec}$ $\mathrm{MeV} /$ nucleon) vs. average magnetic field magnitude for (top) GCR Carbon 100-150 MeV/nucleon from CRIS and (bottom) ACR Oxygen 13-21 MeV/nucleon from SIS. The data have been averaged for 27-day periods. The Pearson's correlation coefficient for the data is shown in each section.

One might expect that the lower rigidity intensities measured by SIS and CRIS are more affected by the 
locally measured magnetic field than the high rigidity intensities (represented by the neutron monitor data). To investigate this, we calculate the ratio of the measured flux to the flux calculated by using the neutron monitor data during the same time period and the power-law fit (i.e. the ratio of each point in Figure 2 and the line). This, in effect, removes from the ACE data any temporal changes in modulation seen by the neutron monitor. In Figure 5 we plot these ratios versus the magnetic field averaged over the same time period. Again, there is no obvious correlation in any of the plots.

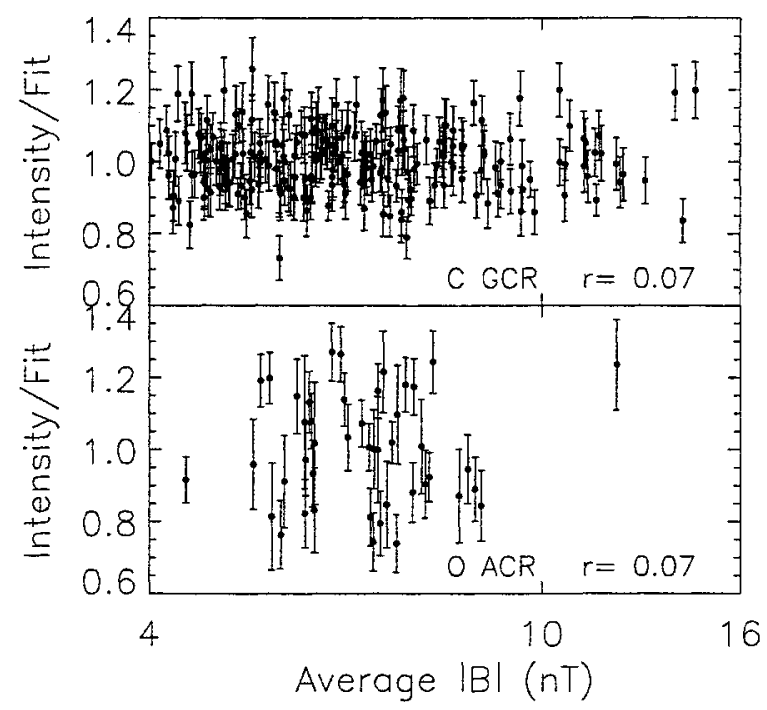

FIGURE 5. Ratio of intensity and Climax neutron monitor fit vs. average magnetic field magnitude for (top) GCR Carbon 100-150 MeV/nucleon from CRIS and (bottom) ACR Oxygen 13-21 MeV/nucleon from SIS. Data in the top panel are daily averages and in the bottom are 7-day averages to improve statistics. The Pearson's correlation coefficient for the data is shown in each section.

\section{CONCLUSION}

This work is still in its early phases. Future work includes better selection of quiet times without biasing the data. Measuring the intensity of the ACRs is becoming more and more difficult as the modulation level increases. It is, however, clear that there are steeper power law indices for the ACR component than for the GCR component (which is to be expected), and the power laws derived here are consistent with those calculated by earlier work.
For this time period, we do not see any correlation between the modulation levels and the magnetic field measured at $1 \mathrm{AU}$. Although this is inconsistent with the observations of (1) and (11), more data is clearly required in order to check this correlation over a larger portion of the solar cycle. Correlation of the particle intensities with the tilt of the neutral current sheet has also been left for the future.

\section{ACKNOWLEDGMENTS}

This research was supported by NASA at the Goddard Space Flight Center, the Jet Propulsion Laboratory, and the California Institute of Technology (under grant NAG5-6912). Climax neutron monitor data are courtesy of the University of Chicago and National Science Foundation grant ATM-9613963. ACE MAG data were supplied by the ACE MAG team through the ACE Science Center.

\section{REFERENCES}

1. Wibberenz, G., et al., Space Sci. Rev., 83, 309 (1998).

2. Stone, E. C., et al., Space Sci. Rev., 86, 357 (1998).

3. Stone, E. C., et al., Space Sci. Rev., 86, 285 (1998).

4. Smith, C. W., et al., Space Sci. Rev., 86, 613 (1998).

5. McDonald, F. B., et al., Astrophys. J., 249, L71 (1981).

6. Burlaga, L. F., et al., J. Geophys. Res., 98, 1 (1993).

7. Mewaldt, R. A., et al., Geophys. Res. Lett., 20, 2263 (1993).

8. Wiedenbeck, M. E., et al., Proc. $26^{\text {th }}$ ICRC (Salt Lake City), 7, 508 (1999).

9. Rygg, T. A., and Earl, J. A., J. Geophys. Res., 76, 7445 (1967).

10. Burlaga, L. F., and Ness, N. F., J. Geophys. Res., 103, 29719 (1998).

11. Cane, H.V. et al., Geophys. Res. Lett., 26, 565 (1999). 